\title{
THIN SETS IN $\mathscr{L}^{1}(S, \mu)$
}

\author{
by I. TWEDDLE
}

(Received 24th August 1970)

\section{Introduction}

In (4) J. F. C. Kingman and A. P. Robertson introduced the idea of thin sets in certain $\mathscr{L}^{1}$ spaces. Thin sets are extreme cases of sets which are not total, and so the problem naturally arises of partitioning a measure space relative to a given set of integrable functions in such a way that on each element of the partition, the set of functions is either thin or total in a sense which is made precise below. In the present note, such partitions are obtained in $\$ 2$ for finite or totally $\sigma$-finite measure spaces. In $\S 3$ the basic ideas are reformulated in terms of Radon measures on locally compact spaces, leading to an extension of the results of $\$ 2$ in this context.

I would like to acknowledge my indebtedness to Professor A. P. Robertson for suggesting this problem to me.

\section{Partitions for finite and totally $\sigma$-finite measure spaces}

Let $(S, \mathscr{M}, \mu)$ be a finite or totally $\sigma$-finite measure space. If $X \subseteq S, \mathscr{M}_{X}$ denotes the $\sigma$-ring $\{Y: Y \in \mathscr{M}, Y \subseteq X\}$. The restriction of $\mu$ to $\left(X, \mathscr{M}_{X}\right)$ will again be denoted by $\mu$. If $M$ is a set of extended real-valued or complex functions on $S, M_{X}$ will denote the set of restrictions of these functions to $X$. The set of positive integers will be denoted by $P$.

Definition 1. Let $M$ be a non-empty subset of $\mathscr{L}^{1}(S, \mu)$ (real or complex), and let $X \in \mathscr{M}$.

(a) $M$ is said to be $X$-thin if for each $Y \in \mathscr{M}_{X}$ with $\mu(Y) \neq 0$, there exists $\phi \in \mathscr{L}^{\infty}(S, \mu)$ such that

(i) $\phi$ is not $(\mu-)$ negligible,

(ii) $\phi$ vanishes off $Y$,

(iii) $\int \phi f d \mu=0$ for all $f \in M$.

If $M$ is $S$-thin, it is said to be thin.

(b) $M$ is said to be $X$-total if the set of equivalence classes of the elements of $M_{X}$ is total in $L^{1}(X, \mu)$.

From the duality of the spaces $L^{1}(S, \mu)$ and $L^{\infty}(S, \mu)$, it follows that $M$ is $X$-thin if and only if for each $Y \in \mathscr{M}_{X}$ such that $\mu(Y) \neq 0, M$ is not $Y$-total (1, Chap. IV, §1, No. 2). The following lemma summarises some consequences of these definitions. The proofs are immediate and are therefore omitted. 
Lemma. (i) If $M$ is $X$-thin (resp. $X$-total) and $Z \subseteq X, Z \in \mathscr{M}$, then $M$ is Z-thin (resp. Z-total).

(ii) If $M$ is $X$-thin (resp. $X$-total) and the symmetric difference of $X$ and $Z$ is negligible, then $M$ is Z-thin (resp. Z-total).

(iii) If $X=\bigcup_{n=1}^{\infty} X_{n}$ and $M$ is $X_{n}$-thin for each $n$, then it is also $X$-thin.

(iv) If $M$ is $X$-thin and $X$-total, then $X$ is negligible.

The next result provides a partition of the type indicated in $\$ 1$.

Theorem 1. Let $(S, \mathscr{M}, \mu)$ be a finite or totally $\sigma$-finite measure space and let $M$ be a non-empty subset of $\mathscr{L}^{1}(S, \mu)$. There exists an at most countable family $\left\{Z_{n}\right\}$ of mutually disjoint elements of $\mathscr{M}$ such that

(i) $M$ is $Z_{n}$-total for each $n$,

(ii) if $Z=\bigcup_{n} Z_{n}, M$ is $(X \mid Z)$-thin for each $X \in \mathscr{M}$.

Proof. Consider first the case in which $\mu$ is finite and so bounded. Proceeding inductively, a sequence $\left(\alpha_{n}\right)$ of real numbers and for each $m \in P$, a sequence $\left(X_{n}^{(m)}\right)$ of elements of $\mathscr{M}$ are constructed such that

(i) $\alpha_{1}=\sup \{\mu(X): X \in \mathscr{M}, M$ is $X$-total $\}$,

(ii) if for each $n \in P, \mathscr{M}_{n}=\left\{X \mid \bigcup_{r=1}^{n} \bigcup_{s=1}^{\infty} X_{s}^{(r)}: X \in \mathscr{M}\right\}$,

$$
\alpha_{n+1}=\sup \left\{\mu(X): X \in \mathscr{M}_{n}, M \text { is } X \text {-total }\right\},
$$

(iii) $M$ is $X_{n}^{(m)}$-total for all $m, n, X_{n}^{(m)} \in \mathscr{M}_{m-1}$ if $m \geqq 2$, and $\mu\left(X_{n}^{(m)}\right) \rightarrow \alpha_{m}$ as $n \rightarrow \infty$ for each $m$.

For each $m, \mu\left(\bigcup_{n=1}^{\infty} X_{n}^{(m)}\right) \geqq \alpha_{m}$, so that

$$
\mu\left(\bigcup_{m=1}^{\infty} \bigcup_{n=1}^{\infty} X_{n}^{(m)}\right)=\sum_{m=1}^{\infty} \mu\left(\bigcup_{n=1}^{\infty} X_{n}^{(m)}\right) \geqq \sum_{m=1}^{\infty} \alpha_{m},
$$

which implies that $\Sigma \alpha_{n}$ converges and consequently, that $\alpha_{n} \rightarrow 0$ as $n \rightarrow \infty$.

Now construct the family

$$
\left\{X_{1}^{(m)}: m \in P\right\} \cup\left\{X_{n+1}^{(m)} \mid \bigcup_{r=1}^{n} X_{r}^{(m)}: m, n \in P\right\} .
$$

Its distinct members form an at most countable collection $\left\{Z_{n}\right\}$ say, of mutually disjoint elements of $\mathscr{M}$, and by part (i) of the lemma, $M$ is $Z_{n}$-total for each $n$. Let $X \in \mathscr{M}$ and let $Z=\bigcup_{n} Z_{n}$. If $M$ is not $(X \backslash Z)$-thin, there exists $Y \in \mathscr{M}_{X \backslash Z}$ such that $\mu(Y) \neq 0$ and $M$ is $Y$-total. Since $Y \in M_{n}$ for each $n$, it then follows that $\mu(Y) \leqq \alpha_{n}$ for each $n$, so that $\mu(Y)=0$. This contradiction shows that $M$ is $(X \backslash Z)$-thin for all $X \in \mathscr{M}$, and completes the proof in the finite case. 
If $\mu$ is a totally $\sigma$-finite measure, then there is a sequence $\left(Y_{n}\right)$ of mutually disjoint elements of $\mathscr{M}$ such that $S=\bigcup_{n}^{\infty} Y_{n}$ and $\mu\left(Y_{n}\right)<\infty$ for each $n$. Since $M_{Y_{m}} \subseteq \mathscr{L}^{1}\left(Y_{m}, \mu\right)$ for each $m$, an at most countable family $\left\{Z_{n}^{(m)}\right\}$ of mutually disjoint elements of $\mathscr{M}$, each contained in $Y_{m}$, can be obtained for each $m$ such that

(i) $M$ is $Z_{n}^{(m)}$-total for each $n$,

(ii) if $W_{m}=\bigcup_{n} Z_{n}^{(m)}, M$ is $\left(X \backslash W_{m}\right)$-thin for each $X \in \mathscr{M}, X \subseteq Y_{m}$.

Enumerate the union of all these families $\left\{Z_{n}^{(m)}\right\}$ as $\left\{Z_{n}\right\}$, and let $Z=\bigcup_{n=1}^{\infty} Z_{n}$. Then clearly $M$ is $Z_{n}$-total for each $n$. If $X \in \mathscr{M}, M$ is $\left(X \cap Y_{m}\right) \backslash W_{m}$-thin for each $m$, so that by part (iii) of the lemma, $M$ is $\bigcup_{m=1}^{\infty}\left\{\left(X \cap Y_{m}\right) \mid W_{m}\right\}$-thin i.e. $M$ is $(X \backslash Z)$-thin, which completes the proof.

Corollary. In the theorem, the family $\left\{Z_{n}\right\}$ can be replaced by a single set $Z \in \mathscr{M}$ if and only if whenever $M$ is $W_{n}$-total, $n \in P$, it is also $\left(\bigcup_{n=1}^{\infty} W_{n}\right)$-total.

Proof. The sufficiency of the condition is immediate from the theorem. Conversely, suppose that there exists $Z \in \mathscr{M}$ such that $M$ is $Z$-total and $M$ is $(X \backslash Z)$-thin for each $X \in \mathscr{M}$. Let $M$ be $W_{n}$-total, $n \in P$. By hypothesis, $M$ is ( $W_{n} \mid Z$ )-thin for each $n$, so that $W_{n} \mid Z$ is negligible by parts (i) and (iv) of the lemma. Thus, since $\bigcup_{n=1}^{\infty} W_{n}=\bigcup_{n=1}^{\infty}\left(W_{n} \cap Z\right) \cup \bigcup_{n=1}^{\infty}\left(W_{n} \mid Z\right)$ and $\bigcup_{n=1}^{\infty}\left(W_{n} \mid Z\right)$ is negligible, it follows from parts (i) and (ii) of the lemma that $M$ is $\left(\bigcup_{n=1}^{\infty} W_{n}\right)$-total.

Remark. The sets $Z_{n}$ in the theorem are not necessarily uniquely determined up to $\mu$-equivalence. However, if $M$ is $X$-thin, $X \cap Z_{n}$ is negligible for each $n$, so that $X \cap Z$ is negligible i.e. $X$ is essentially contained in the complement of $Z$.

\section{Extension to Radon measures}

In this section, Theorem 1 is applied and extended in the case of a positive Radon measure on a locally compact space-for definitions and properties required see (2). In this case $\mathscr{L}^{\infty}(S, \mu)$ is the set of all extended real-valued or complex functions which are bounded locally almost everywhere with respect to $\mu$, and $L^{\infty}(S, \mu)$ is the vector space of equivalence classes in $\mathscr{L}^{\infty}(S, \mu)$ formed by identifying functions which differ only on a locally negligible set. In this way, the duality between $L^{1}(S, \mu)$ and $L^{\infty}(S, \mu)$ is preserved, and consequently it is necessary to reformulate Definition $1(a)$ in this context. The $\sigma$-algebra of all $\mu$-measurable sets is denoted by $\mathscr{H}$. 
Definition 2. Let $M$ be a non-empty subset of $\mathscr{L}^{1}(S, \mu)$ and let $X \in \mathscr{M} . M$ is said to be $X$-thin if for each $Y \in \mathscr{M}_{X}$ which is not locally negligible, there exists $\phi \in \mathscr{L}^{\infty}(S, \mu)$ such that

(i) $\phi$ is not locally negligible,

(ii) $\phi$ vanishes off $Y$,

(iii) $\int \phi f d \mu=0$ for all $f \in M$.

Remark. If $(S, \mathscr{M}, \mu)$ is a (totally) $\sigma$-finite measure space, each locally negligible set is negligible, in which case the two definitions of $X$-thin sets coincide. This will be the case if $S$ is expressible as the union of a sequence of compact sets and so in particular, if $S$ is a separable metrisable locally compact space $(3,3.18 .3)$.

If $M$ is a non-empty subset of $\mathscr{L}^{1}(S, \mu)$, denote by $T(M)$ the union of all open subsets $U$ of $S$ such that $M$ is $U$-thin. Since $T(M)$ is open, it belongs to $\mathscr{M}$.

Theorem 2. Let $\mu$ be a positive Radon measure on a locally compact space $S$ and let $M$ be a non-empty subset of $\mathscr{L}^{1}(S, \mu)$. Then $M$ is $T(M)$-thin.

Proof. Let $Y$ be any subset of $T(M)$ which is an element of $\mathscr{M}$ and which is not locally negligible. Then $Y$ contains an integrable set $W$ with positive measure. Now

$$
\mu(W)=\sup \{\mu(K): K \subseteq W, K \text { compact }\}
$$

and so there is a compact set $K \subseteq Y$ such that $\mu(K)>0$. Each $x \in T(M)$ has an open neighbourhood $U(x)$ such that $M$ is $U(x)$-thin, and since these neighbourhoods form an open covering of $K$, there is a finite collection $U_{r}, 1 \leqq r \leqq n$, say, of such neighbourhoods, whose union contains $K$. It then follows from parts (iii) and (i) of the lemma that $M$ is $K$-thin. Thus there exists $\phi \in \mathscr{L}^{\infty}(S, \mu)$ such that

(i) $\phi$ is not locally negligible,

(ii) $\phi$ vanishes off $K$ and so off $Y$,

(iii) $\int \phi f d \mu=0$ for all $f \in M$.

This shows that $M$ is $T(M)$-thin.

$T(M)$ is therefore the maximal open subset of $S$ on which $M$ is thin. If $S_{1}=S \backslash T(M)$, it is a locally compact space in the topology induced from $S$, being a closed subset of $S$. One might expect to find non-empty open subsets of $S_{1}$ on which $M$ is thin. However, if $U$ is an open subset of $S$ such that $V=U \cap S_{1} \neq \varnothing$ and $M$ is $V$-thin, this, combined with the fact that $M$ is $U \cap T(M)$-thin, shows that $M$ is $U$-thin. Thus $U \subseteq T(M)$, contradicting the assumption that $V \neq \varnothing$.

These ideas are now incorporated in an extension of Theorem 1. 
Theorem 3. Let $\mu$ be a totally $\sigma$-finite positive Radon measure on a locally compact space $S$, and let $M$ be a non-empty subset of $\mathscr{L}^{1}(S, \mu)$. There exists an at most countable family $\{T, Z\} \cup\left\{Z_{n}\right\}$ of mutually disjoint subsets of $S$ such that

(i) $S=T \cup Z \cup \bigcup_{n} Z_{n}$

(ii) $T$ is open and $M$ is $T$-thin,

(iii) there is no open subset $U$ of $S$ such that $U \backslash T \neq \varnothing$ and $M$ is $(U \backslash T)$-thin,

(iv) for each $n, Z_{n}$ is either compact or is the difference of two compact subsets of $S$, and $M$ is $Z_{n}$-total,

(v) $M$ is Z-thin.

Proof. Put $T=T(M)$. Properties (ii) and (iii) then follow from Theorem 2 and the subsequent discussion. Now apply the processes described in the proof of Theorem 1 to the totally $\sigma$-finite measure space $\left(S \backslash T, \mathscr{M}_{S \backslash T}, \mu\right)$ for the subset $M_{S \backslash T}$ of $\mathscr{L}^{1}(S \backslash T, \mu)$. Since $\mu$ has the property that for each integrable set $X$,

$$
\mu(X)=\sup \{\mu(K): K \subseteq X, K \text { compact }\},
$$

it follows from part (i) of the lemma that the sets $X_{n}^{(m)}$ may be chosen to be compact. Now the sets $Z_{n}$ of Theorem 1 are of the form $X_{1}^{(m)}$ or $\left.X_{n+1}^{(m)}\right|_{r=1} ^{n} X_{r}^{(m)}$, so that $Z_{n}$ may be chosen to be compact or the difference of two compact sets. This establishes (iv).

Finally put $Z=S \backslash\left(T \cup \bigcup_{n} Z_{n}\right)$. Since $\mathscr{M}$ is a $\sigma$-algebra, $Z \in \mathscr{M}$ and (i) is satisfied. That $M$ is $Z$-thin now follows from property (ii) of Theorem 1.

In a measure space $(S, \mathscr{M}, \mu)$, a family $\left\{S_{t}: t \in \mathscr{T}\right\}$ of elements of $\mathscr{M}$ is said to be a base for the measure $\mu$ if

(i) each $S_{t}$ has finite measure,

(ii) $S_{r} \cap S_{t}=\varnothing, r \neq t$,

(iii) $\bigcup\left\{S_{t}: t \in \mathscr{T}\right\}=S$,

(iv) for each element $X$ of $\mathscr{M}$ with finite measure, there exists an at most countable union of elements of $\left\{S_{t}: t \in \mathscr{T}\right\}$, which contains all but a negligible subset of $X$.

Although the methods used to obtain the partition of Theorem 3 depend upon the total $\sigma$-finiteness of $\mu$, it is still possible to extract a " maximal" subset on which $M$ is thin if the measure has a base.

Theorem 4. Let $\mu$ be a positive Radon measure on a locally compact space $S$, and let $M$ be a non-empty subset of $\mathscr{L}^{1}(S, \mu)$. If there is a base $\left\{S_{t}: t \in \mathscr{T}\right\}$ for the measure $\mu$, there exists a measurable set $X$ such that $M$ is $X$-thin, and for each set $Z$ such that $M$ is $Z$-thin, $Z \backslash X$ is locally negligible. 
Proof. By Theorem 1, each $S_{t}$ may be partitioned into mutually disjoint measurable sets $S_{t}^{\prime}, Z_{n}^{(t)}, n \in N(t)$, where $N(t)$ is at most countable, such that $M$ is $Z_{n}^{(t)}$-total for each $n \in N(t)$ and $M$ is $S_{t}^{\prime}$ thin. Now put $X=\bigcup\left\{S_{t}^{\prime}: t \in \mathscr{T}\right\}$. If $K$ is any compact subset of $S$, by hypothesis, there exists an at most countable sub-family $\left\{S_{t(n)}\right\}$ of $\left\{S_{t}: t \in \mathscr{T}\right\}$ such that $K_{0}=K \backslash \bigcup_{n} S_{t(n)}$ is negligible. Now

$$
K \cap X=\left(\bigcup_{n}\left(K \cap S_{t(n)}^{\prime}\right)\right) \cup\left(K_{0} \cap \bigcup\left\{S_{t}^{\prime}: t \in \mathscr{T}\right\}\right),
$$

which shows that $K \cap X$ is integrable, since $K \cap S_{t(n)}^{\prime}$ is integrable for each $n$, $K_{0} \cap \bigcup\left\{S_{t}^{\prime}: t \in \mathscr{T}\right\}$ is negligible, and $K \cap X$ is contained in an integrable set. It now follows that $X$ is measurable.

To show that $M$ is $X$-thin, consider any measurable subset $W$ of $X$ which is not locally negligible. Then $W$ contains an integrable set $Y$ of positive measure, and so by hypothesis, there exists $t$ such that $Y \cap S_{t}=Y \cap S_{t}^{\prime}$ has positive measure. Since $M$ is $S_{t}^{\prime}$-thin, there exists $\phi \in \mathscr{L}^{\infty}(S, \mu)$ such that

(i) $\phi$ is not locally negligible,

(ii) $\phi$ vanishes off $Y \cap S_{t}^{\prime}$ and so off $W$,

(iii) $\int \phi f d \mu=0$ for all $f \in M$.

This shows that $M$ is $X$-thin.

Finally let $Z$ be any measurable set such that $M$ is $Z$-thin, and consider $Y=Z \mid X$. If $Y$ is not locally negligible it must contain an integrable set $W$ of positive measure, and as before, there is an at most countable sub-family $\left\{S_{t(n)}\right\}$ of $\left\{S_{t}: t \in \mathscr{T}\right\}$ such that $W=\bigcup\left(W \cap S_{t(n)}\right) \cup W_{0}$ where $W_{0}$ is negligible. Since $M$ is $\left(W \cap S_{t(n)}\right)$-thin and $W \cap S_{t(n)}^{n} \subseteq S_{t(n)} \mid S_{t(n)}^{\prime}$ for each $n$, it follows that $W \cap S_{t(n)}$ is negligible. Thus $W$ is negligible, which gives a contradiction. This completes the proof.

\section{REFERENCES}

(1) N. BouRBAKI, Espaces vectoriels topologiques (Hermann, Paris 1966, 1967).

(2) N. BouRBAKI, Intégration (Hermann, Paris 1965, 1967). 1960).

(3) J. Dieudonnt, Foundations of Modern Analysis (Academic Press, New York

(4) J. F. C. KInGman and A. P. Robertson, On a theorem of Lyapunov, J. London Math. Soc. 43 (1968), 347-351.

UNIVERSITY OF STIRLING

STIRLING 